Original Paper http://ajol.info/index.php/ijbcs http://indexmedicus.afro.who.int

\title{
Contribution à la biométhanisation de la biomasse végétale: cas des résidus de légumes au Burkina Faso
}

\author{
Désiré TRAORE $^{1 *}$, Mahamadi NIKIEMA ${ }^{1}$, Marius K. SOMDA ${ }^{1}$, \\ Joseph B. SAWADOGO ${ }^{1,2}$, Dianou DAYERI ${ }^{3}$ et Alfred S. TRAORE ${ }^{1}$ \\ ${ }^{1}$ Centre de Recherche en Sciences Biologiques Alimentaires Nutritionnelles (CRSBAN), \\ Université de Ouagadougou, O3 BP 7131 Ouagadougou 03, Burkina Faso. \\ ${ }^{2}$ Université Polytechnique de Bobo-Dioulasso, 01 BP 1091 Bobo-Dioulasso, Burkina Faso. \\ ${ }^{3}$ Centre National de la Recherche Scientifique et Technologique (CNRST), \\ 03 BP 7192 Ouagadougou 03, Burkina Faso. \\ *Auteur correspondant, E-mail: desiretraore@gmail.com
}

\section{REMERCIEMENTS}

Nous remercions l'Association BADEYA de France, notamment Joseph Lafitte pour leur appui financier dans la conduite des travaux de recherche.

\section{RESUME}

La matière organique constitue une source de bioénergie par la voie de la digestion anaérobie. Le but de cette étude est de déterminer les paramètres physico-chimiques et les effets de l'inoculation ainsi que de la température sur la biométhanisation des résidus de légumes. Des échantillons de résidus de légumes collectés dans des localités différentes (restaurants universitaires et marchés de légumes) à Ouagadougou ont servi de substrat. L'étude des paramètres physico-chimiques a été réalisé à l'aide des méthodes standard (AOAC, APHA) et le biogaz produit a été analysé par chromatographie en phase gazeuse. Le pH, la matière sèche, la matière sèche volatile, les cendres, le carbone organique, les protéines, les lipides et les carbohydrates totaux ont été respectivement de 5,87; 11,78\%; 80,46\%; 1,3\%; 46,68\%; 10,02\%; 9,95\%; 60,67\%. La composition en minéraux exprimée en mg/g de matière sèche était de $12,69\left(\mathrm{Ca}^{2+}\right) ; 19,44\left(\mathrm{Ka}^{+}\right) ; 11,05\left(\mathrm{Na}^{+}\right) ; 1,608(\mathrm{~N}) ; 3,92$ (p) ; 0,97 $\left(\mathrm{SO}_{4}{ }^{2-}\right)$. L'effet de l'inoculum a été mis en évidence par une production nulle de $\mathrm{CH}_{4}$ à partir d'un temoin non inoculé. La température optimale de production de biométhane a été de $44{ }^{\circ} \mathrm{C}$ avec un rendement de $238 \mathrm{ml} \mathrm{CH} 4 / \mathrm{g}$ de MS.

(C) 2016 International Formulae Group. All rights reserved.

Mots clés : Déchets, légume, valorisation, biogaz, Burkina Faso.

\section{Contribution to the biometanation of plant biomass: case of vegetable residues in Burkina Faso}

\begin{abstract}
Organic material is a source of bioenergy through anaerobic digestion. The purpose of this study is to determine the physico-chemical parameters and the effects of inoculation as well as temperature on biomethanation of vegetable wastes. Samples of vegetable residues collected in different localities (canteens
\end{abstract}


and vegetable markets) in Ouagadougou were used as substrate. The study of physico-chemical parameters was performed using standard methods (AOAC, APHA) and biogas produced was analyzed by gas chromatography. $\mathrm{pH}$, solids, volatile solids, ash, organic carbon, proteins, lipids and total carbohydrates were respectively $5.87 ; 11.78 \% ; 80.46 \% ; 1.3 \% ; 46.68 \% ; 10.02 \% ; 9.95 \% ; 60.67 \%$. The mineral composition expressed as mg/g dry matter was $12.69\left(\mathrm{Ca}^{2+}\right) ; 19.44\left(\mathrm{~K}^{+}\right) ; 11.05\left(\mathrm{Na}^{+}\right) ; 1.608(\mathrm{~N}) ; 3.92(\mathrm{p}) ; 0.97\left(\mathrm{SO}^{2-}\right)$. Inoculum effect was evidenced by no production of $\mathrm{CH}_{4}$ using an uninoculated control. The optimum temperature for biogas production was $44{ }^{\circ} \mathrm{C}$ in a yield of $238 \mathrm{ml} \mathrm{CH} 4 / \mathrm{g} \mathrm{DM}$.

(C) 2016 International Formulae Group. All rights reserved.

Keywords: Waste, vegetables, valorization, Biogas, Burkina Faso.

\section{INTRODUCTION}

Les dernières décennies ont été marquées par une augmentation permanente de la consommation de pétrole dans le monde dont la conséquence est l'épuisement des réserves de combustibles d'origines fossiles et l'augmentation du prix des produits pétroliers. D'autre part, la combustion de combustibles fossiles contribue le plus aux émissions de gaz à effet de serre (GES), qui conduisent à la pollution atmosphérique et au réchauffement climatique (Balat et Balat, 2010). Cela a incité le développement des bioénergies afin de créer des substituts aux énergies d'origines fossiles et d'assurer la protection de l'environnement (McKendry, 2002). Le Burkina Faso n'est pas resté en marge de cette initiative. Plus de 3500 Biodigesteurs sont installés à travers le pays via le Programme National de Biodigesteur (PNB), mais seules les déjections animales sont valorisées. Cependant l'énorme quantité de déchets maraîchers (déchets de légumes) produits chaque année pourrait être utilement exploitée dans ce sens.

En effet, la culture maraîchère occupe une place de choix parmi les filières porteuses retenues par les autorités burkinabè et qui figurent dans le document de stratégie de développement rural à l'horizon 2020. La production annuelle de légume est estimée entre 350000 et 400000 tonnes (Easypol, 2007). Cependant, cette filière présente des handicapes d'autant plus qu'on continue d'enregistrer d'énormes taux de rejets annuels allant de 15 à 20\% (DSA, 2002). Ces pertes sont liées à plusieurs facteurs tels que l'absence de la chaîne de froid, les mauvaises conditions de transport, l'insuffisance des infrastructures routières, l'insuffisance et l'inadéquation des infrastructures de transformation (Judicome, 2004). A ces déchets viennent s'ajouter les résidues émanant des ménages tels que les épluchures et les restes de légume non propices à la consommation humaine. Cette forme de production est quotidienne, pérenne et même universelle. Elle est plus importante dans les institutions telles que les hôtels, restaurants, hôpitaux, écoles et centres de formation, universités, armée, maisons d'arrêt et de correction, centres d'accueil, etc. (Easypol, 2007).

Ces rejets, sous forme de résidus, constituent une nuisance environnementale certaine à prendre en charge pour la sauvegarde durable de notre environnement. Cependant, la matière organique contenue dans ces résidus constitue une source de biogaz par la voie de la digestion anaérobie (Bouallagui, 2004 ; Gunaseelan, 2004; Djaafri et al., 2009).

La digestion anaérobie ou méthanisation est un processus naturel et spontané de biodégradation de la matière organique fermentescible qui s'accompagne de la production de biogaz, melange de méthane et de dioxyde de carbone (Pouch et al., 2005). La production de biogaz et plus précisément de biométhane, permettra le développement durable des zones rurales et des régions enclavées, une diversification des 
ressources énergétiques (Schievano et al., 2009), et la préservation de l'environnement (Ward et al., 2008; Abbasi et Abbasi, 2010).

Le bon fonctionnement du procédé de biométhanisation, est conditioné en grande partie par les conditions physico-chimiques du substrat à traiter et par d'autes paramètres, parmi lesquels l'inoculum et la température qui ont constitué les facteurs étudiés dans la présente étude. En outre, la quantification gazeuse a fait l'objet d'un suivi expérimental tout au long du cycle de production.

Ce travail de recherche vise à apporter une contribution à l'étude des conditions de faisabilité, de développement et d'optimisation de la valorisation des résidus de légumes, à des fins de production d'énergie, de réduction des impacts et nuisances, et de recyclage de la matière par la digestion anaérobie.

Pour y parvenir, la caractérisation physico-chimique des déchets de légumes collectés dans la ville de Ouagadougou et les effets de l'inoculation et de la température sur la productivité de biogaz à partir de ces résidus ont été étudiés.

\section{MATERIEL ET METHODES}

Echantillonnage et traitement des déchets de légumes

La biomasse végétale ayant servi de substrat dans cette étude était composée essentiellement de résidus de légumes, notamment d'épluchures (de concombre, de courgette, d'aubergine, d'oignon bulbe et de pomme de terre) et de feuilles (de choux, d'oignon, de laitue) impropres à la consommation humaine. Ces résidus ont été collectés pendant les mois de janvier à mars 2013 à Ouagadougou au niveau des restaurants universitaires situés dans les secteurs $n^{\circ} 29$ et $n^{\circ} 52 \quad$ (Zogona et Patte d'oie) et dans les marchés de légumes situés dans les mêmes quartiers. Ces échantillons ont été séparément récoltés, séchés au soleil pendant 5 jours. Après séchage, un mélange équitable (masse/masse) de chaque type de déchet a été broyé dans un mortier puis passé au tamis de $1 \mathrm{~mm}$ de diamètre de mailles et la poudre obtenue a été conservée dans des sachets stériles au laboratoire $\left(30 \pm 2{ }^{\circ} \mathrm{C}\right)$.

\section{Analyses physico-chimiques du substrat}

Les analyses des paramètres physicochimiques ont été réalisées en triplicata.

\section{Mesure du pH}

Le $\mathrm{pH}$ a été mesuré sur $2 \mathrm{~g}$ d'échantillon homogénéisés avec $20 \mathrm{ml}$ d'eau distillée à l'aide d'un pH-mètre (WTW pH340) préalablement étaloné avec des solutions de tampon phosphate $(\mathrm{pH} 7)$ et tampon acétate $(\mathrm{pH} 4)$ à $25{ }^{\circ} \mathrm{C}$, selon la méthode de Nout et al. (1989).

\section{Détermination de la matière sèche}

La matière sèche a été déterminée à partir de $5 \mathrm{~g}$ d'échantillons, par pesée différentielle, après passage à l'étuve Heraeus $\mathrm{T} 5042$ à $105^{\circ} \mathrm{C}$ pendant 72 heures (méthode AOAC, 1990).

\section{Matière organique}

Après passage à l'étuve à $105{ }^{\circ} \mathrm{C}$, l'échantillon a été calciné à $600{ }^{\circ} \mathrm{C}$, pendant six heures dans un four à moufle. Ainsi, le pourcentage en matières sèches volatiles a été obtenu par différence entre la mase d'échantillon séchée à $105{ }^{\circ} \mathrm{C}$ et sa masse après calcination à $600{ }^{\circ} \mathrm{C}$ (APHA, 1998).

\section{Carbone organique total}

La teneur en carbone organique a été déduite de la teneur en matière sèche volatile après calcination de l'échantillon dans un four à moufle à $550{ }^{\circ} \mathrm{C}$ pendant deux (2) heures (Alisson, 1965).

\section{Dosage des protéines}

Le taux de proteine a été déterminé par la méthode de Kjeldahl (AOCS, 1990) qui consiste à mineraliser de $0,2 \mathrm{~g}$ d'échantillon en présence de 3,5 $\mathrm{g}$ de sulfate de potassium $\left(\mathrm{K}_{2} \mathrm{SO}_{4}\right)$ et $0,4 \mathrm{~g}$ de sulfate de cuivre $\left(\mathrm{CuSO}_{4}\right)$ ainsi que $10 \mathrm{ml}$ d'acide sulfurique $(\mathrm{HCl})$ concentré suivie d'une distillation en présence de la phénolphtaléine. 


\section{Dosage des lipides}

Les lipides ont une solubilité différentielle dans les solvants organiques. Cette propriété a été utilisée par la méthode de SOXLET pour extraire les lipides contenus dans $5 \mathrm{~g}$ d'échantillon avec l'hexane comme solvant pendant 4 heures (AOAC, 1984).

\section{Dosage des carbohydrates}

Le dosage des carbohydrates a été réalisé par spectrophotométrique selon la méthode de Fox et Robyt (1991). La lecture des densités optiques a été faite à $540 \mathrm{~nm}$ à l'aide d'un lecteur de plaque type $\mu$ quant (Bio-tek instrument $\mathrm{n}^{\circ}$ série 175940 , USA) couplé à un ordinateur muni d'un logiciel KC (v1.31.5) Junior intégré.

\section{Détermination des cendres totales}

Le taux de cendre a été obtenu par pésée, après évaporation de l'éau et l'élimination des constituants organiques par calcination de l'échantillon dans un four à moufle pendant quatre (4) heures selon la méthode de Fouassin et Noirfalise (1981).

\section{Analyse des mineraux}

L'analyse des mineraux a été réalisée dans le laboratoire du Bureau National des Sols (BUNASOL) à Ouagadougou selon la Documentation Technique $\mathrm{N}^{\circ} 3$ BUNASOL (1984).

\section{Dosage du phosphore}

Le phosphore total a été déterminé directement à l'auto-analyseur Skallar après minéralisation de l'échantillon à l'aide de l'acide sulfurique $\left(\mathrm{H}_{2} \mathrm{SO}_{4}\right)$ et du $\mathrm{C}_{7} \mathrm{H}_{6} \mathrm{O}_{3}$ en présence d'eau oxygénée $\left(\mathrm{H}_{2} \mathrm{O}_{2}\right)$.

\section{Dosage des ions $\mathrm{Na}^{+}$et $\mathrm{K}^{+}$}

Les ions $\mathrm{Na}^{+}$et $\mathrm{K}^{+}$ont été déterminé par la méthode de photomètre à flamme (spectrophotomètre CORNNING 400) après une minéralisation de l'échantillon à l'aide d'une solution mixte d'acide sulfuriquesélénium-salicylique.

\section{Dosage des ions $\mathrm{Ca}^{2+}$}

L'échantillon a été soumis à une minéralisation avec un melange d'acide nitrique et chloridrique concentré. Après traitement au nitrate de lanthane, la teneur en $\mathrm{Ca}^{2+}$ a été déterminée par spectromètrie d'absorption atomique.

\section{Dosage des ions sulfates}

Les ions sulfates ont été déterminés par spectrophotomètrie d'absoption après minéralisation

\section{Processus de digestion anaérobie Prélèvement et traitement de l'inoculum}

L'ajout d'un inoculum d'ensemencement lors du démarrage des tests de biométhanisation, met à disposition une biomasse microbienne active qui permet d'éviter des cas d'inhibition liés en particulier à une accumulation d'acides gras volatils (AGV) et une baisse de $\mathrm{pH}$, et favorise un état d'équilibre de l'ensemble du processus de digestion anaérobie.

Des résidus de panse prélévés dans les panses de bœufs fraichement abattus à l'abattoir frigorifique de Ouagadougou ont servi d'inoculum dans notre étude. Une fois au laboratoire, ces résidus ont été dilués $(\mathrm{m} / \mathrm{v}$, 4/5) avec une solution tampon constituée de 2 $\mathrm{g}$ de $\mathrm{K}_{2} \mathrm{HPO}_{4}+2 \mathrm{~g}$ de $\mathrm{NH}_{4} \mathrm{Cl} / 1000 \mathrm{ml}$ d'eau distillée pour empêcher une chute du $\mathrm{pH}$. L'inoculum ainsi traité a été distribué dans des flacons de $500 \mathrm{ml}$ sous flux d'azote qui ont été ensuite placés sous agitation dans un incubateur Shaker (Innova ${ }^{\text {TM }} 4000$, U.S.A) pendant une heure. Après cette phase d'homogéinisation, les flacons contenant l'inoculum ont été séparément incubés pendant 10 jours respectivement à température ambiante $\left(28-30^{\circ} \mathrm{C}\right), 37{ }^{\circ} \mathrm{C}$ et à $44^{\circ} \mathrm{C}$ avant le démarrage des expériences, afin d'épuiser la matière organique de départ. De ce fait, le gaz produit était quotidiennement dégagé à l'aide d'une seringue. Ces résidus de panses ont été ensuite acclimatés dans les mêmes conditions de température d'incubation sur le substrat d'étude par une série de cinq transferts successifs (chaque 21 jours), puis la 
production de biogaz a été suivie par chromatographie en phase gazeuse (CPG).

Etude de l'effet de l'inoculation sur la biométhanisation des résidus de légumes

Des flacon septum de $120 \mathrm{ml}$ ont servi de reacteurs en mode batch selon la méthode de Angelidaki et al. (2009).

Le tampon phosphate composé de $2 \mathrm{~g}$ de $\mathrm{K}_{2} \mathrm{HPO}_{4}+2 \mathrm{~g} \mathrm{NH}_{4} \mathrm{Cl}$ pour $1000 \mathrm{ml}$ d'eau distillée a été utilisé comme milieu de base (Milaiti et al., 1998) afin d'empêcher la chute de $\mathrm{pH}$ lors des expériences. Le milieu réactionnel constitué de milieu tampon et de l'échantillon a été reparti dans les flacons selon une charge organique de $2 \%$. Après 10 à 15 minutes d'agitation manuelle, les flacons ont été légèrement fermés (préfermentation aérobie). Au bout de 72 heures de préfermentation, le $\mathrm{pH}$ a été mesuré, ajusté autour de 7,0 avec du $\mathrm{NaOH} 10 \mathrm{~N}$ et l'inoculum a été ensuite ajouté dans un rapport $\mathrm{v} / \mathrm{v}$ de $10 \%$. Les flacons ont été enfin fermés hermétiquement pour garantir une étanchéité parfaite aux gaz. L'anaérobiose totale dans le milieu a été réalisée ensuite par dégazage sous flux d'azote après l'inoculation pendant 1 à 2 minutes. Les tests ont été réalisés en triplicatat et des tests témoins sans inoculum ont été réalisés dans les mêmes conditions à $37{ }^{\circ} \mathrm{C}$ afin de rendre compte de l'effet de l'inoculum.

Etude de l'effet de la température sur la biométhanisation des résidus de légumes

L'effet de la température sur la digestion anaérobie de ces résidus a été réalisé selon la même méthodologie décrite ci-dessus, sauf que les cultures ont été toutes inoculées avec la bouse de vache et incubés à $44{ }^{\circ} \mathrm{C}$, $37^{\circ} \mathrm{C}$ et $30^{\circ} \mathrm{C}$ respectivement.

\section{Analyse chromatographique des gaz produits \\ L'analyse du biogaz a été réalisée par CPG à l'aide de l'appareil Girdel serie 30 à catharomètre, muni d'un détecteur à conductibilité thermique, équipé d'un}

enregistreur potentiomètrique SERVOTRACE type sefram Paris $1 \mathrm{~mm}$. Le chromatographe a été équipé de deux colonnes remplies Porapak Q80/100 (pour doser le $\mathrm{CH}_{4}$ ) et Porapak Q100/120 (pour doser le $\mathrm{CO}_{2}$ ) montées en parallèle et programmé comme suit: température de l'injecteur $90{ }^{\circ} \mathrm{C}$, température du four $60{ }^{\circ} \mathrm{C}$, température du détecteur 100 ${ }^{\circ} \mathrm{C}$, pressions du gaz vecteur hydrogène 1 bar (pour doser le $\mathrm{CO}_{2}$ ) et 2 bars (pour doser le $\mathrm{CH}_{4}$ ), courant filament $150 \mathrm{~mA}$ et atténuation 16. Ainsi, $0,5 \mathrm{ml}$ de la phase gazeuse dans chaque flacon a été prélévé et injecté à travres les colonnes du CPG. Vingt quatre (24) flacons ont servi pour chaque test et à chaque analyse, trois (3) flacons étaient sacrifiés pour mesurer le $\mathrm{CH}_{4}$ et $\mathrm{CO}_{2}$ produits dans chaque cas. Les teneurs en $\mathrm{CH}_{4}$ et $\mathrm{CO}_{2}$ ont été déterminées à l'aide de courbes étalons établies à patir de $\mathrm{CH}_{4}$ et $\mathrm{CO}_{2}$ standards.

\section{Courbes d'étalonnage de $\mathrm{CO}_{2}$ et $\mathrm{CH}_{4}$}

A partir du témoin $\mathrm{CO}_{2}$ pur, produit par Burkina Industrial Gas (bouteille de $30 \mathrm{~kg}$ $\mathrm{CO}_{2}$; volume $50 \mathrm{~L}$ et pureté $99 \%$ ), des volumes de 0,$2 ; 0,3 ; 0,4 ; 0,5$ et $0,6 \mathrm{ml}$ ont été injectés dans le Girdel afin d'obtenir des réponses (aires des pics du $\mathrm{CO}_{2}$ ) qui ont permis d'établir l'équation suivante de la courbe de régression:

Volume $\mathrm{CO}_{2}(\mu \mathrm{l})=0,0137 \times$ Aire du pic $\mathrm{CO}_{2}+0,0074\left(\mathrm{r}^{2}=0,9992\right)$

Le méthane étalon a été synthétisé avec une pureté de $90 \%$ dans un flacon de $50 \mathrm{ml}$. Comme précédemment, différents volumes $(0,1 ; 0,2 ; 0,3 ; 0,4 ; 0,5$ et $0,6 \mathrm{ml})$ de ce gaz ont été injectés afin d'obtenir l'équation suivante de la courbe de régression:

Volume CH4 $(\mu \mathrm{l})=13,308 \times$ Aire du pic de CH4 +1,1753 $\left(r^{2}=0.9994\right)$.

\section{Analyses statistiques}

Toutes les données ont été soumises à une analyse de variance (ANOVA) avec le logiciel XLSTAT- PRO 7.5.2 et les résultats ont été comparés en utilisant le test de Fisher au seuil de probabilité $\mathrm{p}=5 \%$. 


\section{RESULTATS}

Caractéristiques physico-chimiques des résidus de légumes

Les données des paramètres physicochimiques des résidus de légumes sont consignées dans le Tableau 1.

Le Tableau 1 montre que le substrat a un $\mathrm{pH}$ acide $(5,87 \pm 0,23)$ avec une teneur en matière séche de $11,78 \pm 0,6 \%$. Sa teneur en matière organique est assez importante compte tenu de la composition en proteines $(10,02 \pm 0181 \%)$, lipides $(9,95 \pm 0,234 \%)$ et en hydrates de carbone $(60,67 \pm 0,67 \%)$. Les valeurs ioniques sont de $12,69 \%\left(\mathrm{Ca}^{2+}\right)$; $19,44 \%\left(\mathrm{Ka}^{+}\right) ; 11,05 \%\left(\mathrm{Na}^{+}\right) ; 1,608 \%(\mathrm{~N})$; $3,92 \%(\mathrm{P}) ; 0,97 \% \quad\left(\mathrm{SO}_{4}{ }^{2-}\right)$. Les rapports carbone/azote $(\mathrm{C} / \mathrm{N})$ et carbone/phosphore $(\mathrm{C} / \mathrm{P})$, beaucoup déterminant dans la détermination des paramètres de biométhanisation d'un substrat ont été de 29 , 03 et 119,09 respectivement pour $\mathrm{C} / \mathrm{N}$ et $\mathrm{C} / \mathrm{P}$.

\section{Effet de l'inoculation et de la période sur la production de $\mathrm{CH}_{4}$ et $\mathrm{CO}_{2}$}

Les résultats de l'analyse de variance des productions moyennes de $\mathrm{CH}_{4}$ et $\mathrm{CO}_{2}$ en fonction de l'inoculation et de la période d'incubation sont présentés dans le Tableau 2.

Il réssort que l'inoculation et la période d'incubation ont un effet très significatif respectivement sur les productions de $\mathrm{CH}_{4}$ et $\mathrm{CO}_{2}(\mathrm{p}<0,0001)$. Il apparait également que les effets combinés de l'inoculation et de la période influent très significativement sur la production du biogaz $(\mathrm{p}<0,0001)$ comme présenté dans le Tableau 2.

L'étude de la cinétique de production de biogaz en fonction de l'inoculum a montré une production importante de $\mathrm{CO}_{2}$ dès les prémiers jours de digestion anaérobie en général. Cependant, cette production de $\mathrm{CO}_{2}$ a diminué à partir du $8^{\text {ème }}$ jour de 23763,78 à $19332,06 \mu 1$ le $13^{\text {ème }}$ jour et a chuté progressivement jusqu'à $17808,66 \mu 1$ le $38^{\text {ème }}$ jour en présence d'inoculum. Par contre, dans le cas du témoin non inoculé, la production de $\mathrm{CO}_{2}$ a augmenté légèrement de $2100,11 \mu \mathrm{l}$ le $4^{\text {ème }}$ jour jusqu'à atteindre $26000 \mu \mathrm{L}$ le $23^{\text {ème }}$ jour et est devenue presque constante jusqu'à la fin de la digestion.

L'analyse chromatographique de la composition gazeuse a révélé une différence de production de $\mathrm{CH}_{4}$ hautement significative $(\mathrm{p}<0,0001)$ entre l'échantillon inoculé et l'échantillon témoin non inoculé (Figure 2). Cette figure présente une production nulle de $\mathrm{CH}_{4}$ au niveau du témoin (sans inoculum) durant toute la période d'incubation. Par contre, une production moyenne de 22537,98 $\mu 1$ de $\mathrm{CH}_{4}$ a été obtenue dans le cas de l'échantillon inoculé.

\section{Effet de la température et de la période sur la production de $\mathrm{CH}_{4}$ et $\mathrm{CO}_{2}$}

Des résultats de cette étude (Tableau 3), il ressort que la température et la période ainsi que leur effets combinés ont tous un effet très significatif sur la production de biogaz ( $\mathrm{p}<0,0001$ ).

La Figure 2 montre que la production de $\mathrm{CO}_{2}$ est importante pendant les premiers jours de fermentation $\left(30162,72 \mu \mathrm{l}\right.$ à $44{ }^{\circ} \mathrm{C}$, $23104,04 \mu \mathrm{l}$ à $37^{\circ} \mathrm{C}$ ) et est également plus prononcée au $13^{\text {ème }}$ jour à $44^{\circ} \mathrm{C}$ et $37^{\circ} \mathrm{C}$ par rapport à celle obtenue à $30^{\circ} \mathrm{C}(21201 \mu \mathrm{l})$.

La Figure 2 présente également une relation inverse entre la production de $\mathrm{CO}_{2}$ et la formation du $\mathrm{CH}_{4}$. Elle montre qu'au fur et à mesure que les pics de $\mathrm{CO}_{2}$ diminuent, ceux du $\mathrm{CH}_{4}$ augmentent.

Des analyses du biogaz produit, il ressort qu'aux températures de $44^{\circ} \mathrm{C}$ et $37^{\circ} \mathrm{C}$, la production du $\mathrm{CH}_{4}$ est relativement rapide par rapport à sa production à $30{ }^{\circ} \mathrm{C}$ tout comme la production et la consommation du $\mathrm{CO}_{2}$.

En outre, au bout de 38 jours de biométhanisation, le rendement en méthane dans les réacteurs à $44{ }^{\circ} \mathrm{C}$ était plus important $\left(238 \mathrm{ml} \mathrm{CH} / \mathrm{g}\right.$ MS) que celui à $37^{\circ} \mathrm{C}(214,8$ $\mathrm{ml} \mathrm{CH4/g} \mathrm{MS} \mathrm{qui} \mathrm{se} \mathrm{trouve} \mathrm{également} \mathrm{plus}$ élévé que celui à température ambiante (180 $\mathrm{ml} \mathrm{CH} 4 / \mathrm{g} \mathrm{MS}$ ). 


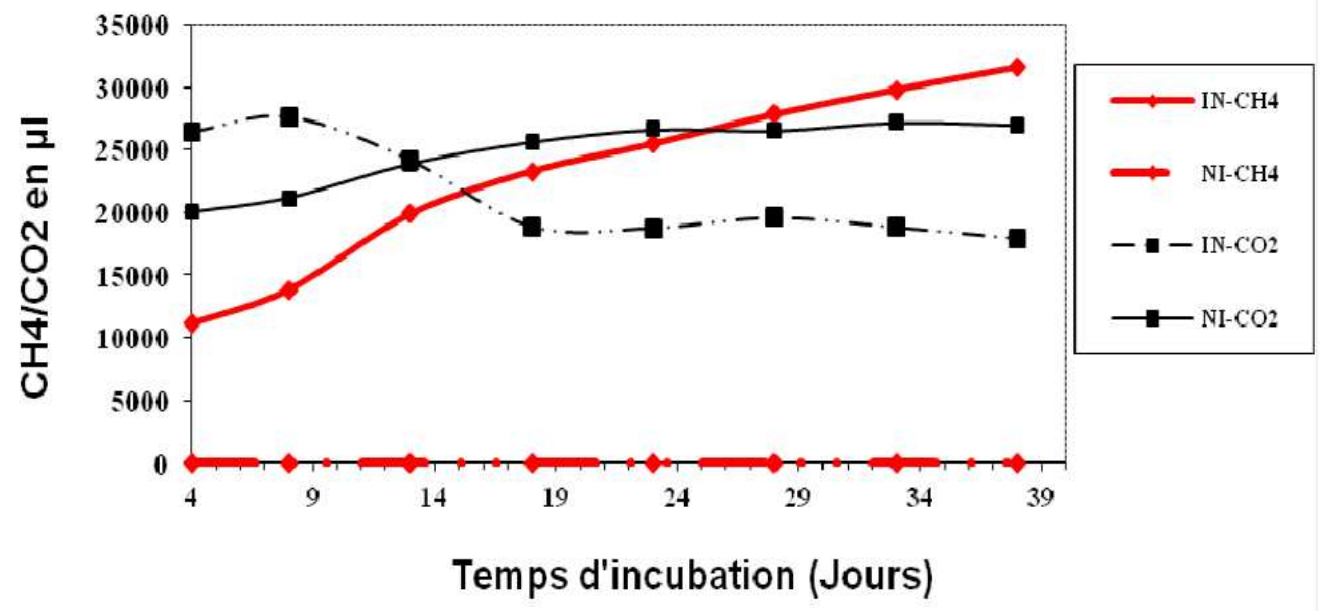

Figure 1: Cinétique de production de $\mathrm{CH}_{4}$ et de $\mathrm{CO}_{2}$ en fonction de l'inoculum et du temps d'incubation.

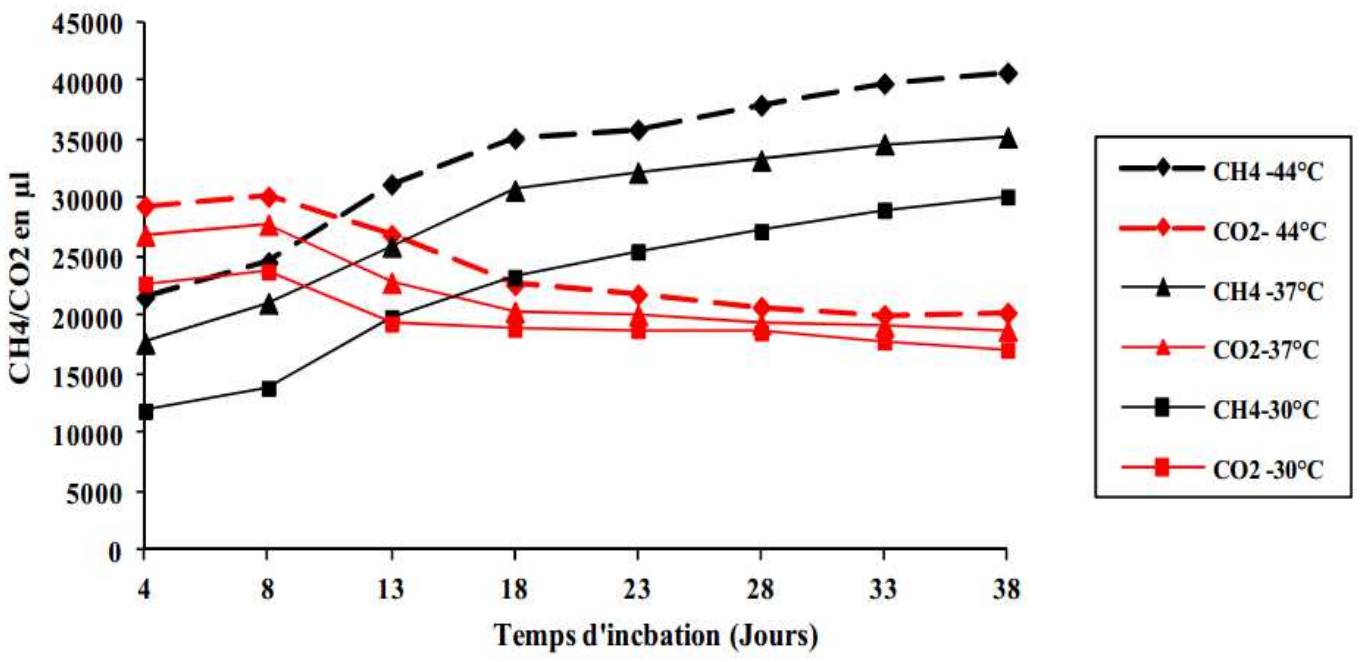

Figure 2: Cinétique de production de $\mathrm{CH}_{4}$ et de $\mathrm{CO}_{2}$ en fonction de la température et du temps d'incubation. $\mathrm{CH}_{4}-44^{\circ} \mathrm{C}=$ courbe de $\mathrm{CH}_{4}$ à $44^{\circ} \mathrm{C} ; \mathrm{CO}_{2}-44^{\circ} \mathrm{C}=$ courbe de $\mathrm{CO}_{2}$ à $44{ }^{\circ} \mathrm{C} ; \mathrm{CH}_{4}-37^{\circ} \mathrm{C}=$ courbe de $\mathrm{CH}_{4}$ à 37 ${ }^{\circ} \mathrm{C} ; \mathrm{CO}_{2}-37{ }^{\circ} \mathrm{C}=$ courbe de $\mathrm{CO}_{2}-37{ }^{\circ} \mathrm{C} ; \mathrm{CH}_{4}-30{ }^{\circ} \mathrm{C}=$ courbe de $\mathrm{CH} 4$ à $30{ }^{\circ} \mathrm{C} ; \mathrm{CO}_{2}-30{ }^{\circ} \mathrm{C}=$ courbe de $\mathrm{CO}_{2}-30{ }^{\circ} \mathrm{C}$ 
Tableau 1: Paramètres physicochimiques du substrat d'étude.

\begin{tabular}{|c|c|}
\hline Paramètres & Valeurs \\
\hline $\mathrm{pH}$ & $5,87 \pm 0,23$ \\
\hline Matière sèche \% (p/ps) & $11,78 \pm 0,6$ \\
\hline Matière organique $\%(\mathrm{p} / \mathrm{ps})$ & $80,46 \pm 0,021$ \\
\hline -lipides \% (p/ps) & $9,95 \pm 0,234$ \\
\hline -protéines \% (p/ps) & $10,02 \pm 0,181$ \\
\hline -Carbohydrates $\%(\mathrm{p} / \mathrm{ps})$ & $60,67 \pm 0,67$ \\
\hline Cendres \% (P/Ps) & $1,36 \pm 0,06$ \\
\hline -Phosphore (mg/g MS) & $0,392 \pm 0,12$ \\
\hline$-\mathrm{Na}^{+}(\mathrm{mg} / \mathrm{g} \mathrm{MS})$ & $11,05 \pm 0,028$ \\
\hline$-\mathrm{K}^{+}(\mathrm{mg} / \mathrm{g} \mathrm{MS})$ & $19,446 \pm 0,03$ \\
\hline$-\mathrm{Ca}^{2+}(\mathrm{mg} / \mathrm{g} \mathrm{MS})$ & $12,69 \pm 0,29$ \\
\hline$-\mathrm{SO}_{4}^{2-}(\mathrm{mg} / \mathrm{g} \mathrm{MS})$ & $0,97 \pm 0,1$ \\
\hline -Azote $(\mathrm{N})(\mathrm{mg} / \mathrm{g} \mathrm{MS})$ & $1,608 \pm 0,024$ \\
\hline Carbone organique (p/ps) & $46,686 \pm 0,007$ \\
\hline $\mathrm{C} / \mathrm{N}$ & 29,033 \\
\hline $\mathrm{C} / \mathrm{P}$ & 119,09 \\
\hline
\end{tabular}

Tableau 2 : Analyse de variance de la production de $\mathrm{CH}_{4}$ et de $\mathrm{CO}_{2}$ en fonction de l'inoculum et de la période d'incubation.

\begin{tabular}{|c|c|c|c|c|c|}
\hline \multirow[t]{3}{*}{ Source de variation } & ddl & \multicolumn{4}{|c|}{$\operatorname{Biogaz}(\mu \mathrm{l})$} \\
\hline & & \multicolumn{2}{|c|}{$\mathrm{CO}_{2}$} & \multicolumn{2}{|c|}{$\mathrm{CH}_{4}$} \\
\hline & & $\mathbf{F}$ & $\mathbf{P}$ & $\mathbf{F}$ & $\mathbf{P}$ \\
\hline Inoculum & 2 & 187,193 & $<0,0001 * *$ & 441,739 & $<0,0001 * *$ \\
\hline Période & 8 & 22,449 & $<0,0001 * *$ & 6,439 & $<0,0001 * *$ \\
\hline Inoculum*Période & 16 & 203,308 & $<0,0001 * *$ & 6,439 & $<0,0001 * *$ \\
\hline
\end{tabular}

Tableau 3: Analyse de variance de la production moyenne de $\mathrm{CH}_{4}$ et de $\mathrm{CO}_{2}$ en fonction de la température et de la période d'incubation.

\begin{tabular}{|c|c|c|c|c|c|}
\hline \multirow[t]{3}{*}{ Source de variation } & \multirow[t]{3}{*}{ ddl } & \multicolumn{4}{|c|}{$\operatorname{Biogaz}(\mu \mathrm{l})$} \\
\hline & & \multicolumn{2}{|c|}{$\mathrm{CO}_{2}$} & \multicolumn{2}{|c|}{$\mathrm{CH}_{4}$} \\
\hline & & $\mathbf{F}$ & $\mathbf{P}$ & $\mathbf{F}$ & $\mathbf{p}$ \\
\hline Température & 2 & 86,714 & $<0,0001 * *$ & 39,976 & $<0,0001 * *$ \\
\hline Période & 8 & 69,240 & $<0,0001 * *$ & 46,720 & $<0,0001 * *$ \\
\hline Température*Période & 16 & 4,386 & $<0,0001 * *$ & $\mathbf{0 , 5 0 8}$ & $<0,0001 * *$ \\
\hline
\end{tabular}

$\mathrm{F}=\mathrm{F}$ de Fischer $; *$ significatif au seuil $1 \%$. 


\section{DISCUSION}

\section{Paramettres physico-chimiques des résidus de légumes}

Le $\mathrm{pH}$ acide du substrat $(5,87 \pm 0,23)$ est dû à la présence d'acide organique dans les différents déchets. Charnay (2005) a montré que le $\mathrm{pH}$ des suspensions de déchets solides varie entre 5 et 9 .

La détermination de la matière sèche (MS) est un des critères qui permet de classer le substrat en fonction de son aptitude à être plus ou moins dégradable par voies biochimiques. Elle conditionne le choix thermochimique (pyrolyse, gazéification) pour les teneurs en matière sèche supérieures à $30 \%$, ou biochimique par fermentation, pour des teneurs inferieures comme dans le cas précis dans cette étude. Moletta (2008) rapporte que la teneur en matière sèche (MS) $\mathrm{du}$ substrat à méthaniser détermine généralement le choix des procédés de méthanisation et le type de digesteur anaérobie. La teneur en matière sèche du substrat d'étude $(11,78 \pm 0,6)$ nous confronte à une digestion anaérobie par « voie humide » qui est adaptée au traitement des déchets ayant entre 5 et $20 \%$ de taux de matière sèche (MS).

La composition du substrat en protéines, lipides et en hydrate de carbone lui confère les caractéristiques d'un substrat de prédilection pour les microorganismes de la fermentation anaérobie et prédispose la biomasse végétale à une biodégradation importante.

Les faibles teneurs en protéine et lipide du substrat sont favorables à sa méthanisation d'autant plus que, à forte concentration, les lipides, notament les acides gras à longue chaîne, peuvent former une barrière autour de la matière organique empéchant ainsi l'accès aux microorganismes (Neves et al., 2008). En ce qui concerne les protéines, au cours de la digestion anaérobie, ils sont transformés en ammoniac qui est présent sous forme libre/non ionisée $\mathrm{NH}_{3}$ ou sous forme ionisée $\mathrm{NH}_{4}$ (ammonium). A faible concentration, l'ammoniac (une base forte) peut neutraliser les acides gras volatils produits lors de l'acidogénèse, ce qui aide à maintenir un $\mathrm{pH}$ neutre, favorable à la digestion anaérobie. En revanche, à forte concentration, l'ammoniac est toxique pour les bactéries impliquées dans la méthanisation Buffiere et al. (2007).

Les valeurs ioniques du substrat $(12,69$ $\left(\mathrm{Ca}^{2+}\right) ; 19,44\left(\mathrm{Ka}^{+}\right) ; 11,05\left(\mathrm{Na}^{+}\right) ; 1,608(\mathrm{~N})$; $\left.3,92(\mathrm{p}) ; 0,97\left(\mathrm{SO}_{4}^{2-}\right)\right)$ ne sont pas limitantes et peuvent théoriquement stimuler sa digestion anaérobie. La littérature montre que des concentrations d'ions $\mathrm{Na}^{+}, \mathrm{K}^{+}$et $\mathrm{Ca}^{2+}$ peuvent devenir inhibitrices puis toxiques à des valeurs élévées. He et al. (2006) ont démontré que le sodium et le potasium ont un effet inhibiteur sur l'hydrolyse et l'acidogénège des déchets d'origine végétale à des concentrations de 25 à $50 \mathrm{~g} / \mathrm{l}$.

Quant au soufre, sa présence à un certain niveau de concentration crée une compétition entre les bactéries sulfatoréductrices et les bactéries méthanogènes pour l'acceptation des électrons. Il est aussi connu que les substrats riches en protéines soufrées et en sulfates peuvent mener à une augmentation de la production de $\mathrm{H}_{2} \mathrm{~S}$ qui est toxique pour les microorganismes méthanogènes (Bernet et Buffière, 2008). La valeur en sulfate du substrat d'étude $(0,97 \%)$ est suffisament faible et ne peut donc pas gener sa méthananogénèse.

Les rapports $\mathrm{C} / \mathrm{N}, \mathrm{C} / \mathrm{P}$, permettent de prévoir d'une manière générale l'état d'équilibre général influençant la digestibilité d'un substrat. Les études d'Ostrem en 2004 ont montré que la proportion désirable se situe entre 20 et 30, 25 étant le ratio idéal pour le $\mathrm{C} / \mathrm{N}$ et 150 pour le $\mathrm{C} / \mathrm{P}$ (Ostrem, 2004). Ces rapports sont de 29,03 et 119,09 respectivement pour $\mathrm{C} / \mathrm{N}$ et $\mathrm{C} / \mathrm{P}$ et sont proches des valeurs optimales qui sont de 30 pour $\mathrm{C} / \mathrm{N}$, et 150 pour $\mathrm{C} / \mathrm{P}$ selon la littérature (Ostrem, 2004). 


\section{Effet de l'inoculation et de la période sur la production de $\mathrm{CH}_{4}$ et $\mathrm{CO}_{2}$}

La différence de production de $\mathrm{CH}_{4}$ hautement significative $(\mathrm{p}<0,0001)$ présentée dans le Tableau 2 s'expliquerait par un blocage de processus de méthanisation en absence d'inoculum du fait de plusieurs raisons: La préfermentation peut être une cause d'inhibition dans la mésure où elle accelère la vitèsse de l'hydrolyse du substrat (Kirtane et al., 2009). En effet, en présence de substrats solubles ou rapidement hydrolysables, les bactéries acidogènes à développement rapide et tolérantes aux $\mathrm{pH}$ acides vont générer d'importantes quantités d'acide gras volatiles (AGV), abaissant le $\mathrm{pH}$. Ces derniers devront être consommés au fur et à mesure de leur production par les bactéries acétogènes et méthanogènes afin de ne pas atteindre des valeurs de $\mathrm{pH}$ critiques. Le développement lent de ces bactéries est succeptible de bloquer totalement le processus de biodégradation comme suite: plus d'acidité entrâne une baisse de $\mathrm{pH}$ qui se traduit par une diminution de l'activité des bactéries utilisant ces acides avec pour concéquence plus d'acides cumulés. Cet enchaînement peut conduire à acidifier le milieu jusqu'au point où plus aucune bactérie ne peut se développer (Batstons et al., 2002).

Vavilin et al. (2003) ont montré que la méthanogène nécessite un équilibre entre formation et consommation d'acides. Cet équilibre est désigné sous le nom de balance acidogène/méthanogène. Cela pourrait expliquer l'accumulation de $\mathrm{CO}_{2}$ dans le cas non inoculé où cet équilible est bouleversé.

En outre, le temps d'incubation qui a été de 38 jours, peut s'avérer insuffisant compte tenu du ralentissement de la digestion anaérobie dans les digesteurs non ensemencés.

Djaafri et al. (2009) ont montré que dans le cas de digestion anaérobie sans apport d'inoculum comme source microbienne (cas non ensemencé), le déclenchement de la phase de méthanogénèse n'est possible qu'à partir du $55^{\text {s̀me }}$ jour. Dans cette étude, il a été révélé que le $\mathrm{pH}$ se stabilise aux environs de 6,5 pendant 50 jours et augmente à nouveau dès le $60^{\text {ème }}$ jour pour atteindre la valeur de 7,7 à la fin de l'expérience (phase méthanogène).

Ces résultats permettent de confirmer que l'apport d'inoculum au substrat favorise nettement la digestion anaérobie et accelerait le développement des bactéries méthanogènes. L'inoculation (ensemenssement) apporte un consortium de microorganismes qui interviennent dans les différentes phases de dégradation de la matière organique.

\section{Effet de la température et de la période sur la production de $\mathrm{CH}_{4}$ et $\mathrm{CO}_{2}$}

La relation inverse entre la production de $\mathrm{CO}_{2}$ et la formation du $\mathrm{CH}_{4}$ peuvent s'expliquer par les points suivants:

-Le fait que la diminution de la production de $\mathrm{CO}_{2}$ soit couplée avec l'augmentation de la production de $\mathrm{CH}_{4}$ indique clairement la reduction du $\mathrm{CO}_{2}$ en $\mathrm{CH}_{4}$ par les bactéries méthanogènes hydrogénophiles en présence d'hydrogène moléculaire. Il a été démontré que dans les milieux anoxiques, le $\mathrm{CO}_{2}$ est réduit par l'hydrogène $\left(\mathrm{H}_{2}\right)$ en $\quad \mathrm{CH}_{4}$ par les méthanogènes et aussi par les sulfatoréductrices en présence du sulfate et par les homoacétogènes (Garcia et al., 2000).

-La production intense de $\mathrm{CO}_{2}$ (Figure 2) durant les premiers jours de fermentation suggère que les trois prémières phases (hydrolyse, acidogénèse, acétogènèse) de la méthanisation sont effectivement fonctionnelles dans les prémiers jours de la méthanisation.

Bouallagui et al. (2004) ont démontré que le rendement en biométhane est plus élevé dans les reacteurs thermophiles par rapport aux reacteurs mésophiles et aux réacteurs psychrophiles de $41 \%$ et $144 \%$, respectivement. Les rendements de biométhane obtenus dans notre étude s'inscrivent dans la plage du potentiel 
biométhanogène des déchets organiques ménagés selon la littérature pour les températures de $44{ }^{\circ} \mathrm{C}$ et $37{ }^{\circ} \mathrm{C}$. Par contre, le rendement obtenu à $30{ }^{\circ} \mathrm{C}$ est légèrement inférieur à cette plage. Cette plage de potentiel biométhanogène varie de 200 à $600 \mathrm{ml}$ de $\mathrm{CH} 4 / \mathrm{g}$ de matière sèche selon Trazié (2007) et $265 \mathrm{ml} / \mathrm{g}$ de matière sèche selon Servais (2008).

Cependant, le faible rendement à $30{ }^{\circ} \mathrm{C}$ peut s'expliquer par l'insuffisance du temps de digestion d'autant plus que les faibles températures ralentissent l'activité métabolique des bactéries méthanogènes. Il a été démontré que plus la température de digestion anaérobie est élévée, plus la productivité gazeuse est importante et le processus de production plus rapide (Gunaseelan, 2004). Kim et al. (2006) ont trouvé que la production de biogaz selon la température s'établit comme suit : $30^{\circ} \mathrm{C}<35$ ${ }^{\circ} \mathrm{C}<40{ }^{\circ} \mathrm{C}<45{ }^{\circ} \mathrm{C}$. De même, Gunaseelan (2004) a montré que la cinétique de conversion des déchets de fruits et légumes en biométhane était plus élevée à $35^{\circ} \mathrm{C}$ qu'à 28 ${ }^{\circ} \mathrm{C}$. Ces paramètres traduisent donc le fait que l'augmentation de la température d'incubation permet d'accroître considerablement les activités métaboliques des microorganismes partenaires de la méthanisation et par conséquent le rendement de la production de biométhane.

\section{Conclusion}

Les donnés de l'analyse des paramètres physico-chimiques des déchets de légume ne montrent aucun paramètre majeur contraignant leur bioconversion en biogaz. La production optimale de biométhane a été obtenue à $44^{\circ} \mathrm{C}$ et en présence d'inoculum.

L'ensemble des résultats constitue un acquis majeur pour la valorisation énergétique des déchets de légumes par biométhanisation. C'est donc une source de biomasse végétale à prendre en compte dans la mise en place d'une politique de recherche de source d'énergies nouvelles et renouvelables pour un devéloppement durable dans un pays comme le Burkina Faso.

\section{CONFLIT D'INTERET}

Les auteurs déclarent qu'ils n'ont aucun conflit d'intérêts.

\section{CONTRIBUTIONS DES AUTEURS}

DT a réalisé les travaux de laboratoire et a rédigé le manuscrit ;

DD a suivie les travaux de recherche, a participé à la lecture et la correction du manuscrit; AST a dirigé les travaux; MKS a participé à la lecture et la correction du manuscrit; JBS et MN ont participé aux travaux de laboratoire.

\section{REMERCIEMENTS}

Nous remercions Oscar OUEDRAOGO pour sa collaboration lors de la collecte des échantillons au niveau des restaurants universitaires. Nous remercions Pr Aboubakar S. OUATTARA, Dr Aly SAVADOGO, Dr Checkna ZONGO pour leurs contributions dans ce travail.

\section{REFERENCES}

Abbasi T, Abbasi SA. 2010. Production of clean energy by anaerobie digestion of phytomass-New prospects for a global warming amelioration technology. Renew Sustain Energy Rev., 14(6): 1653-1659. DOI :10.1016/j.rser.2010.03.003

Allison LE. 1965. Methods of soil analysis. Am. Soc. Agron., Madison, Wisconsin: 1367-1378.

American Public Health Association (APHA). 1998. Standard Methods for the Examination of Water and Wastewater, 20th ed. APHA, Washington, DC. Anaerobic Ecosystem. Adv. Microbiol. Ecol. USA., 3: 49-77.

Angelidaki M, Alves D, Bolozonella L, Borazacconi JL, Campos AJ, Guwy S, Kalyuhnyi P, Jenicek, Van JB. 2009. 
Defining the biomethan potential (BMP) of solid organic wastes and energy crops: a proposed protocol for batch assays. Water Science \& Technology, 59: 927934. DOI: 10.2166/wst.2009.040

AOCS (American Oil Chemists Society). 1990. Official methods and recommended pratics $\left(4^{\text {th }}\right.$ edn $)$. American Oil Chemists Society.

Association of Official Analytical Chemists (AOAC). 1984. Official Methods of Analysis (14 ${ }^{\text {th }}$ edn). AOAC: Washigton DC, USA; 144.

Balat M, Balat H. 2010. Progress in Biodiesel Processing. Applied Energy, 87 (5): 18151835. DOI: $\quad 10.1016 /$ j.apenergy. 2010.01.012

Batstone DJ, Keller J, Angelidaki I, Kalyuzhnyi S, Pavlostathis SG, Rozzi A, Sanders W, Siegriest H, Vavilin VA. 2002. The IWA anaerobic digestion model $\mathrm{N}^{\circ} 1$ (ADM1). Water Science end Technology, 45(10): 65-73.

Bernet N, Buffière P. 2008. La méthanisation. In Caractérisation de la Mise en CEuvre de la Méthanisation, Moletta R. (ed). Éditions Tec \& Doc : Paris ; 87-113.

Bouallagui H, Haouari O, Touhami Y, Cheikh BR, Marouani L, Hamdi M. 2004. Effect of temperature on performance of an anaerobic tubular reactor treating fruit and vegetable waste. Process Biochemistry, 39: 2143-2148. DOI: 10.1016/j.procbio.2003.11.022

Buffiere P, Carrere M, Lemaire O, Vasquez J. 2007. Guide méthodologique pour l'exploitation d'unités de méthanisation de déchets solides, Projet MethapiExpertise, P. 40.

Charnay F. 2005. Compostage des Déchets Urbains dans les Pays en Développement: Elaboration d'une Démarche Méthodologique pour une Production Pérenne de Compost. Thèse de Doctorat, Université de Limoges, p. 229.
Djaafri M, Khelifi S, Kalloum A, Tahri K, Touzi A. 2009. Effet de l'ensemencement sur la digestion anaérobie des déchets ménagers de la ville d'Adar. Revue des Energies Renouvelables, 12 (3): 369-374.

Documentation Technique $\mathrm{N}^{\circ} 3$ Bureau National des Sols (BUNASOL). 1984. Méthodes d'analyse physiques et chimiques des Sols, Eaux et Plantes, Burkina Faso, p. 133.

Easypol 107. 2007. Analyse de la filière maraîchage au Burkina Faso. Ressources pour l'élaboration des politiques, 112 pages.

Fouassin A, Noirfalise A. 1981. Méthodes d'Analyse des Substances Alimentaires (4 ${ }^{\text {ème }}$ édn). Presses Universitaires, Université de Liège, Faculté de Médecine ; 19-35.

Fox JD, Roxbyt JF. 1991. Minuarization of tree carbohydrates analysis using a microsample plate reader. Anal. Bioch. 195: 93-96.

Gunaseelan V. 2004. Biochemical methane potential of fruit and vegetable solid waste feedstocks. Biomass and Bioenergy, 26: 389-399. DOI:10.1016/j.biombioe.2003.08.006

He PJ, LÜ F, Shao LM, Pan XJ, Lee DJ. 2006. Effect of alkali metal cation on the anaerobic hydrolysis and acidogenesis of vegetable waste. Environmental Technology, 27(3): 317-327. DOI: $10.1080 / 09593332708618646$

Jingquan L. 2006. Optimization of anaerobic digestion of sewage sludge using thermophilic anaerobic pre-treatment. BioScience and Technology, BioCentrum-DTU, Technical University of Denmark DK-2800: Lyngby Denmark; 59.

Judicome. 2004. Etude pour l'élaboration du plan de développement de la filière fruits et légumes/Rapport final janvier, p. 164 .

Kim JK, Oh BR, Chun YN, Kim SW. 2006. Effects of Temperature and Hydraulic 
Retention Time on Anaerobic Digestion of Food Waste. Journal of Bioscience and Bioengineering, The Society for Biotechnology, Japan, 102(4): 328-332. DOI: $10.1263 /$ jbb.102.328

Kirtane RD, Suryawanshi PC, Pati MR, Chaudhari, Kothari. 2009. Optimization of organic loading rate for different fruit waste during biomethanization. Journal of Scientific \& Industrial Reaserch, 68: 252-255.

Mckendry P. 2002. Energy Production from Biomass (Part 1): Overview of Biomass, Bioresource Technology, 83: 37-46.

Milaiti M. 1998. Thèse de doctorat: Etude des paramètres de biofermentation de Calotropis procera en vue de la conception et de la mise en œuvre d'un pilote de fermentation méthanique à partir de cette biomasse. Université de Ouagadougou, 198 pp.

Moletta R. 2008. La Méthanisation. Editions Tec \& Doc: Paris : 532.

Neves L, Goncalo E, Oliviera R, Alves MM. 2008. Influence of composition on thebiomethanisation potential of restaurant waste at mesophilic temperature. Waste Management, 28(6): 965-972. DOI: 10.1016/j.wasman.2007. 03.031

Nout MJR, Rombouts FM, Havelarr A. 1989. Effect of accelrated natural lactic fermentation of infant food ingredients on some pathogenic microorganisms. Int. J. Food Microbiol., 8: 351-361. DOI:10.1016/j.fm.2014.05.016

Ostrem K. 2004. Greening Waste: Anaerobic digestion for treating the organique fraction of municipal solid waste.
Mémoire de maitrise, Columbia University, New York, p. 59.

Pouch P, Coudure R, Marcato CE. 2005. Intérêt de la Co-digestion pour la vororisation des lisiers et le traitement de déchets fermentescibles à l'échelle d'un territoire. Journées Recherche Porcine, 37: 39-44.

SChievano A, D'imporzano G, Adani F. 2009. Substituting energy crops with organic wastes and agro-industrial residues for biogas production. Journal of Environnment Management, 90: 25372541. DOI:10.1016/j.procbio.2009.03. 014

Servais C. 2008. La Méthanisation. Edition Tec Doc Lavoisier : Paris, 7430-1036.

Trazie G, Irje BI. 2007. Etude technique d'une unité de biométhanisation destinée à l'électrification rurale: Application au monastère de keur moussa. Mémoire de diplôme d'ingénieur de conception, Université Check Anta Diop de Dakar, $110 \mathrm{p}$.

Vavilin VA, Rytov SV, Lokhina LY, Pavlostathis SG, Barlaz MA. 2003. Distributed model of solid waste anaerobic digestion, effects of leachate recirculation and $\mathrm{pH}$ adjustement. Wiley Periodicals, 81: 66-73. DOI: 10.1002/bit.10450

Ward AJ, Hobbs PHJ, Holliman PJ, Jones DL. 2008. Optimisation of the anaerobic digestion of agricultural ressources. Bioressource Technology, 99: 7928-7940. 